\title{
Innovation Education Research Based on Cross-object Interactive Robot Simulation Platform
}

\author{
Lei Cheng, Shichao Guo*, and Jin Wu \\ School of Information Science and Engineering, Wuhan University of Science and Technology, \\ Wuhan 430081, China
}

\begin{abstract}
China's robot virtual experiment simulation education experiment teaching service platform was successfully constructed, it is an important pioneer and demonstrator of virtual experiment teaching. It is combined with the superior subject resources of the Robot Engineering major of Wuhan University of Science and Technology. This paper explains the necessity of cross-object interactive robot simulation experiment teaching. The experimental project of the industrial robot simulation module is combined with the teaching of related courses, the teaching system of the cross-object interactive robot virtual simulation experiment teaching platform is analysed. It is proposed that the virtual simulation platform can play a good role in innovating teaching resources in universities and cultivating innovative and entrepreneurial talents in universities.
\end{abstract}

\section{Introduction}

As we all know, practical teaching in today's era has become vital to the cultivation of talent. The Ministry of Education issued the "Notice on Carrying out the Construction of the National Virtual Simulation Experimental Teaching Cent", it is required to continuously promote the in-depth integration of robotics and advanced experimental teaching. Higher education experimental teaching quality and practical education level need to be improved. Various universities have conducted many experimental studies.

In order to actively respond to the current social development to cultivate innovative talents, this paper is to meet the new requirements of the Ministry of Education's teaching reform. This paper is guided by the teaching content of "interactive robot simulation experiment". With modern network technology, a cross-object, interactive, and diversified virtual simulation experiment teaching platform has been developed. The teaching content of virtual simulation experiment has been further improved. The experiment broadens the depth and space of virtual simulation experiment teaching. This paper provides better learning conditions for innovative robot engineering talents who cultivate solid robot basic knowledge, strong practical skills and innovative capabilities. [1-3]

\footnotetext{
* Corresponding author: 846707116@qq.com
} 


\section{Cross-object interactive robot simulation platform design}

\subsection{Necessities and deficiencies of robot simulation platform teaching}

The interactive virtual simulation platform constructed in this paper is mainly the interaction between students and computers. "Cross-object interactive robots" virtual simulation experiment courses mainly include robot modelling, motion planning, decisionmaking control, etc., because the actual robot is expensive, the difficulty of operation makes it difficult for students to master the robot in a short time, it's also difficult to conduct a large number of experiments in a short period of time. Therefore, a virtual simulation experiment platform suitable for robot teaching is needed to further deepen and consolidate what students have learned in class. Through continuous simulation experiments, students can better understand the robot model and have a better grasp of robot motion control. Continuously improve students' hands-on ability and independent innovation ability, and at the same time solve the problem of incomplete robot hardware and inability to carry out practical experiments. [4, 5]

At present, many Robots virtual simulation software have been developed in the market, but only for teaching, these soft wares have the following problems: The software design is complicated to be easy for students to use; There is a certain deviation between software content and teaching content; It is difficult to reflect robot motion characteristics in software. [6]

This paper addresses the shortcomings of the aforementioned robot virtual simulation experiment. Based on Ros software, this research group independently developed a virtual simulation experiment platform suitable for course teaching in colleges and universities. This platform uses open teaching, which not only provides help for school students, but also provides an experimental platform for robot enthusiasts. Users can freely create robotrelated models in the simulation environment. Moreover, the platform supports cross-object research, and students can simulate and control the path planning, movement obstacle avoidance and other operations of different types of robots in the same environment.

\subsection{Innovation education mechanism with simulation platform}

With the development of science and technology, the virtual simulation experiment platform has become an important content of education informatization to drive the modernization of education, which can significantly promote the reform and innovation of teaching content and teaching mode. Whether the simulation platform can simulate the real environment more realistically, to a certain extent, it affects the training of students' problem-solving ability. Most of the robot simulations on the market are based on MATLAB simulation, or Mobile Sim simulation software from Mobile Robot. The running speed of the former is too slow, the latter is incomplete for many physics features, and lacks the physics engine at the same time. This paper is based on the ROS robot virtual simulation technology, and makes full use of the simplicity and environmental scalability of the Ros robot simulation platform. Sensors have been used to obtain environmental information, and technologies such as robot modeling, motion control, path planning, and optimization algorithms have been designed. Students experience the characteristics of interactive robots through the experimental results of 3D models. By building multiple sensors, students can conduct multiple robot simulation experiments across objects in the same environment. Simulation experiments can give full play to students' innovative method research and practical application learning, and cultivate students' independent innovation ability. 


\subsection{Architecture of cross-object robot virtual simulation experiment platform}

Students interact with the simulation platform through the universal interface API. As shown in the figure, the communication layer is divided into two parts: sensor and actuator. The first function of the sensor is to separate the interface from the simulation environment. The collected information is sent to students for use through software processing, so that the control logic program can switch between different robots to operate in the same operating environment. Another function of the sensor is to interact with the virtual environment to obtain information such as the model and location in the real parameters, and then transmit it to the virtual environment. The role of the actuator is similar to that of the sensor. The actuator responds to the student's control program, then generates instructions to change the robot's behavior. The communication layer encapsulates the external communication interface, performs the analysis, packaging and distribution of message packets, adopts adaptation technology, and controls the physical robot through user code. The simulator provides a simulation experiment function for the entire system. This robot virtual simulation platform adopts a client-server architecture. The simulator can be regarded as a server and a virtual robot as a client, which better reflects the characteristics of interaction. In order to realize the innovative feature of cross-object, a number of sensors and actuators were built. Students can complete simulation experiments of multiple robots in the same environment. [7]

\section{Virtual simulation experiment application based on Ros robot}

\subsection{Simulation model construction and optimization}

There are two ways for students to build a robot simulation model based on Ros: The first is to write a URDF model file directly with code to describe the characteristics of the model. URDF file is a special xml file format, which is a descriptive file, usually used for the creation of robot models. In this paper, building a robot model through URDF files requires a certain understanding of the concepts of each part. The first is link and joint, the connection between link is realized through joint, it is similar to the existence of robot joints. The appearance of the link also needs to be configured with version information and form information. In this way, the link is not the existence of a mass point, so the expression of the link really exists. Need three-dimensional rotation coordinate information, as well as the rotation coordinate information of each dimension, that is, the deflection around the $\mathrm{x}$-axis, the pitch around the y-axis, and the z-axis. The deflection information here is described in radians. The second is to build the shape of the robot model through 3D drawing software, and then export it into a URDF file. [8-10]

The XACRO file is a model file after optimizing the URDF file, solved the problems of URDF file with lengthy code, excess of duplicate content, and inconvenience to modify model file parameters, etc. In addition, XACRO has a parameter calculation function, which is more conducive to secondary development. [11]

\subsection{Gazebo scene construction}

First open the Gazebo software through the terminal, execute the Ros launch command in the command to open Gazebo instead of opening it directly. Then students can create the required simulation environment by themselves. There are two ways for students to create. The first is to add a simulation environment model from the list of simulation models provided to users by Gazebo. As shown in Figure 1, users can add and create their own 
simulation environment according to their own needs. As shown in Figure 2, if the simulation model in the insert list cannot meet the needs of students, it is also possible to complete the design of the environment model through 3D design software and load it into the environment list. [12, 13]

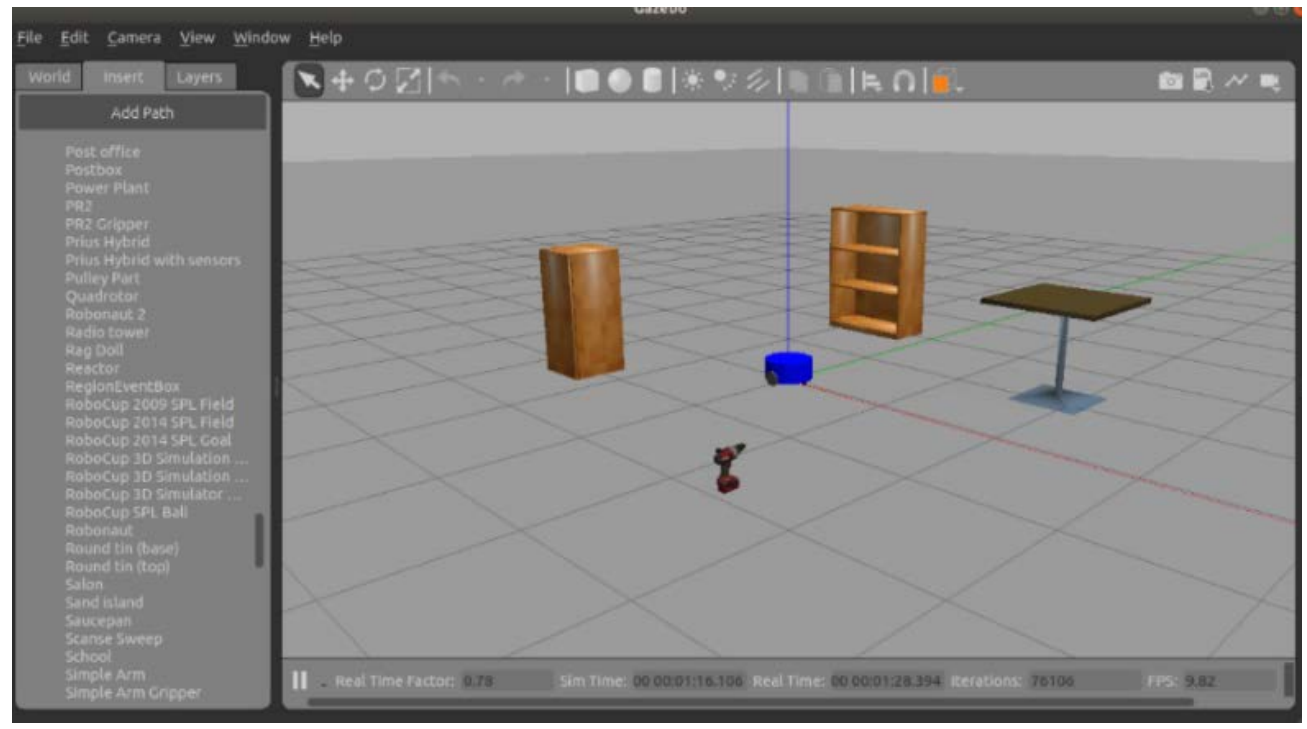

Fig. 1. Add simulation model

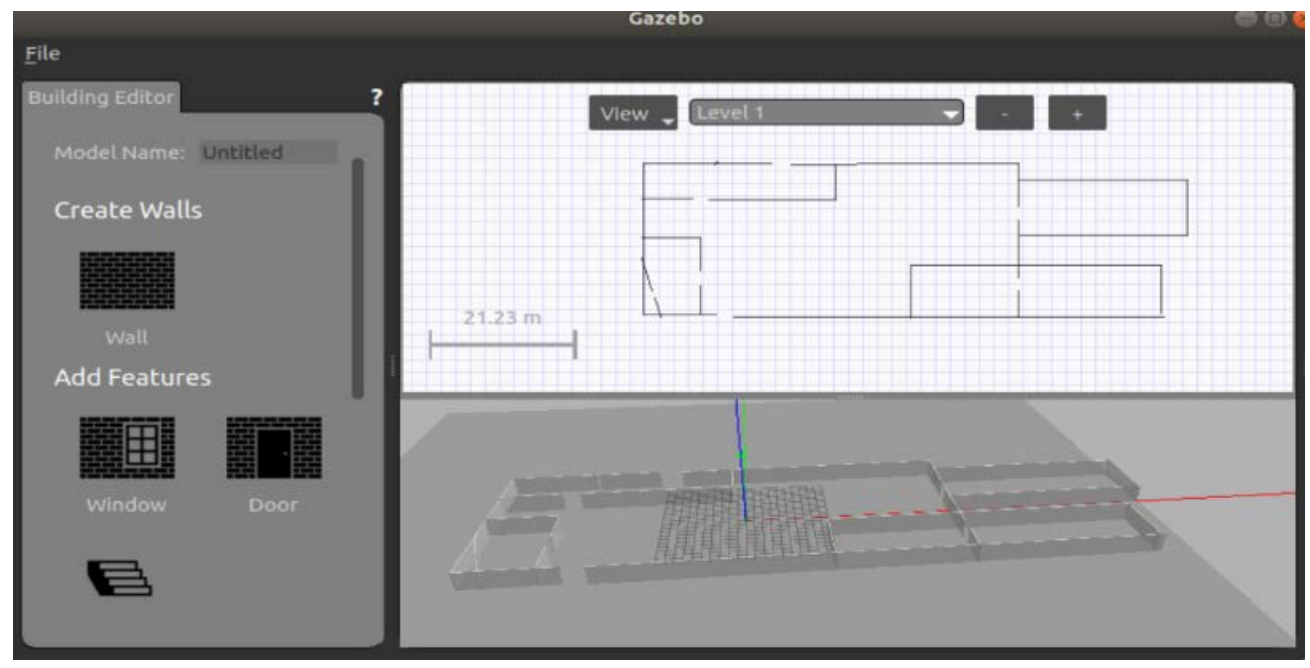

Fig. 2. Edit Building Editor

\subsection{Robot virtual simulation realization}

After logging on to the platform, when students want to simulate a robot, they must first establish a robot model, and then configure a controller to control their robot. As shown in Figure 3, this experiment established a two-wheeled mobile robot. The two-wheeled mobile robot performs two-wheel differential control, so the differential controller is used to 
control the movement of the robot. First, configure the coordinate system of the car body, because of the differential speed control, the distance between the two wheels should be configured in the file so that the differential speed controller can calculate the differential speed. Then configure the frequency of control. The next step is to configure the various parameters of the PID controller. PID is a commonly used control method in control. Students use PID control to quickly reach the desired speed of the wheel. Finally, the upper limit of the wheel acceleration is configured, so that the movement of the mobile robot can be more stable and the performance can be better. After the controller is configured, students can import algorithms and try to do some robot simulation experiments for path planning. [14, 15]

After students have built a mobile robot, they can use the same method to build robotic arms and multi-legged robots in the same environment. The "cross-object" of innovative education in this article is thus realized. Students can perform simulation experiments on the robot through the simple operation of ROS, which greatly improves the characteristics of human-computer interaction, and better completes the homework assigned by the teacher to the students, so that the students have a deeper understanding of the robot, thereby improving the overall teaching quality.

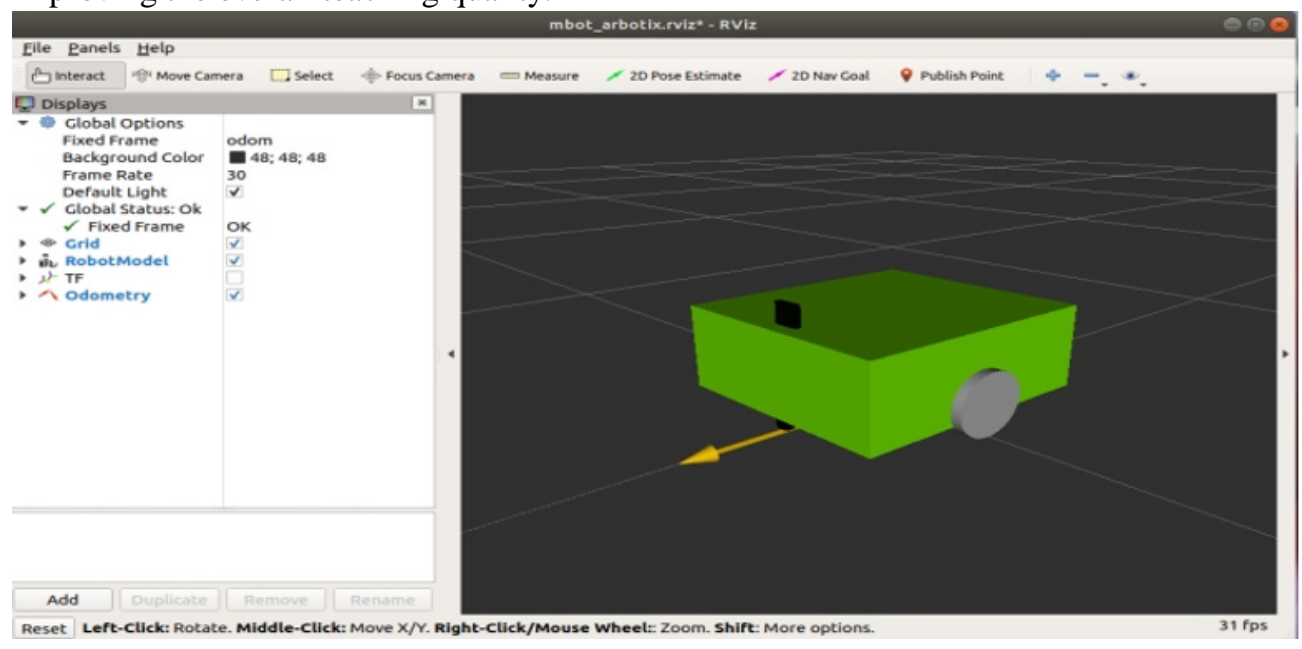

Fig. 3. Mobile robot controlling process

\section{Features and innovations of virtual simulation}

This paper was aimed at the experimental teaching need of robotics courses. The crossobject interactive robot virtual simulation platform described in this article; it has the following characteristics:

The first feature is interactive. The interaction of this robot experimental platform is reflected in the interaction between students and robot simulation. This improves the usability of the platform. Due to the interactive system, it's easier for students to complete robot experiments independently in class, aroused the enthusiasm of the students. Effectively communicate with teachers when encountering problems. Teachers can effectively grasp the learning situation of students through communication, thereby improving the overall teaching quality.

The second feature is comprehensive. The robot virtual simulation platform contains a variety of experimental problems involved in robot teaching. Such as kinematic model, sensor model, motion control, trajectory tracking, path planning, etc. Experiments can 
effectively guide students to better grasp the basic principles, basic models, information processing, decision-making control and other issues of robots, so as to lay a solid foundation for students' future robot learning.

The third feature is cross-object. The innovation of virtual simulation technology in this paper is mainly reflected in the simulation environment of cross-object robots. Students no longer need to build multiple simulation environments to simulate different robot simulations. [16] Different robot models, such as robotic arms, mobile robots, multi-legged robots, etc., can be constructed simultaneously in a simulation environment. This innovation allows students to more intuitively feel the specification parameters of different robot structural models. Moreover, the simulation in the same environment saves time and effort, which greatly improve the learning efficiency.

\section{Conclusions}

This paper mainly focuses on the cross-object interactive robot virtual simulation teaching platform as the research object. First, this paper states the necessity and current shortcomings of the mobile robot virtual simulation teaching experiment system, and also reviewed the innovation education of robot simulation platform. Then build the robot virtual simulation model and environment. The design of a cross-object, interactive simulation platform was elaborated in detail. Through the cross-object virtual simulation experiment mode, students can compare different models of manipulators, mobile robots, and multi-legged robots in the same environment. This is the embodiment of innovative education in this article. This article is a comprehensive practical education system aimed at cultivating students' interest, independence and innovation ability. Cross-object interactive robot simulation platform can also play a role in radiation. More students can participate in learning about robot-related knowledge, thereby it has good social benefits.

\section{References}

1. Shuo Wang. Research progress of robotics [J], Acta automatic Sinical, 2016, 39(7): 963-972.

2. Hanfang Chu, Yankong Zhu. Practice and exploration of entrepreneurship virtual simulation experiment teaching center [J]. Experimental Technology and Management, 2020(12): 97-100.

3. MUBINO, STEVENSCJ, SHAHIDS, et al. A review of the applicability of robots in education[J]. Journal of Technology in Education and Learning, 2018, 1(209-0015): 17.

4. MILLERDP, NOURBAKHSHI. Robotics for education[M] // Springer Handbook of Robotics. Berlin, Germany: Spring Verlag, 2019: 2115-2134.

5. TOHLPE, CAUSOA, TZUOPW, et al. A review on the use of robots in education and young children[J]. Journal of Educational Technology \& Society, 2018, 19(2): 148-163.

6. Hongqi Xiong. New era teaching characteristics of the national virtual simulation experiment teaching project[J]. Experimental Technology and Management, 2019,36(9): 1-4.

7. Lixin Yang, Lianghui Huang. Discussion on Virtual Simulation Integration Technology Based on Robot Experimental Teaching [J]. Laboratory Research and Exploration, 2017, 36(11): 103-106, 125. 
8. Suyun Ge. Motion simulation platform for mobile robots [D]. Xi'an: Xi Dian University, 2019.

9. Guchao Wang. Design and Research of Omnidirectional Mobile Robot System Based on ROS [D]. Anhui: Anhui University of Science and Technology, 2019.

10. Yongping Chen, Yongyan He, Kaikai Wang. Research on ROS-based model construction and optimization of robotic arm [J]. Microcomputer Applications, 2020, 36(2): 13-16.

11. 11. Liang Yang, Yu Fu, etc. Real-time robot based on ROS Development of an innovative experimental platform for positioning and map construction [J]. Experimental technology And Management, 2017, 34 (008): 99-103.

12. Feng Li. Design of Robotic Arm Control System Based on ROS [J]. Automatic Chemical Technology and Application, 2018, 37 (11): 76-80.

13. Jingyang Wu. Research on Multi-Autonomous Mobile Robot System Based on Lidar Positioning and Navigation Harbin Institute of Technology, 2017.

14. Xiang Li. Research on the Navigation Technology of Indoor Mobile Robot Based on SLAM [D]. Harbin Institute of Technology, 2018.

15. Xuansong Chu. Positioning and navigation technology of indoor mobile robots [J]. China New Communications, 201820 (02): 73.

16. Yanling Xia, Xia Yao, Zhongshui Liang. Teaching research based on industrial robot virtual simulation system[J]. Technology Wind, 2020(15): 37. 